\title{
REVISTA DEL CENTRO DE ESTUDIOS CONSTITUCIONALES
}

SUMARIO DEL NUM. 14 (enero-abril 1993)

\section{SEMINARIOS DEL CENTRO DE ESTUDIOS CONSTITUCIONALES}

LUIS AGUILAR DE LUQUE: Los límites de los derechos fundamentales.

ENOCH AlBERTI ROVIRA: La cláusula de libre circulación y la garantía de la unidad del mercado interno en la Constitución Española de 1978.

Manuel Ballestero: Acerca de «lo político».

Hugo E. Biagint: Identidad y educación en la Argentina.

Demetrio Castro Alfin: Godwin y las paradojas de la igualdad.

Juan Francisco Garcia Casanova: Del racionalismo armónico al pragmático: clave bermenéutica del poder real del institucionismo krausista.

Humberto NJaim: Invitación a las Obras completas

Alfonso Rurz Mrguel: Autonomia individual y derecho a la propia vida (un análisis filosófico-jurídico).

2. ESTUDIOS PREMIADOS POR EL C.E.C

3. DOCUMENTACION

Boletin de sumarios.

Bibliografia.

4. ACTIVIDADES DEL CENTRO DE ESTUDIOS CONSTITUCIONALES

PRECIOS DE SUSCRIPCION ANUAL

España

4.500 ptas.

Extranjero

$59 \$$

Número suelto: España

1.600 ptas.

Número suelto: Extranjero

$20 \$$

Pedidos y suscripciones:

CENTRO DE ESTUDIOS CONSTITUCIONALES

Fuencarral, 45 - 28004 MADRID

CENTRO DE ESTUDIOS CONSTITUCIONALES

Plaza de la Marina Española, 9

28071 MADRID 


\title{
REVISTA DE ESTUDIOS POLITICOS
}

\author{
(NUEVA EPOCA) \\ PRESIDENTE DEL CONSEJO ASESOR: Carlos OlLERo GOMEZ \\ DIRECTOR: Pedro de Vega GaRcía \\ SECRETARIO: Juan J. SOLOZABAL ECHEVARRIA \\ SUMARIO DEL NUM. 79 (enero-marzo 1993)
}

\section{ESTUDIOS}

Peter HABERLE: Derecho constitucional común europeo.

Gonzalo FERNANDEZ DE LA MoRA: El proceso contra el Padre Mariana.

Joaquín VARELA SUANZES: Un precursor de la monarquía parlamentaria: Blanco-Wbite y «el español» (1810-1814).

Giacomo SANI: Ciudadanos y sistema político: Participación y cultura política de masas en Italia.

Cesáreo R. Aguilera de Prat y Jaume Vernet Llovet: Cuestiones simbólicas y Constitución española.

Elvyra Elena Pabon Tarantino: Colombia y su revolución pacífica. La nueva Constitución del 5 de julio de 1991. Inicio de un marco institucional dentro de un contexto político pluralista.

\section{NOTAS}

Juan Luis Perez Francesch: Notas acerca del gobierno como órgano de dirección política en la Constitución española de 1978. En especial su incidencia en el ejercicio de las potestades normativas.

H. C. F. MANSILLA: Apogeo y declinación del movimiento sindical boliviano 1982-1985. Una nota sobre la cultura política del autoritarismo.

Arsenio GINZO FERNÁNDEZ: La religión civil y el pensamiento político de Rousseau

Manuel SANCHEZ DE Dios: El modelo sueco de Estado de bienestar.

\section{CRONICAS Y DOCUMENTACION}

Francisco FeRnÁndeZ SEGADO: La correlación entre el tamaño de las circunscripciones y las distorsiones de la proporcionalidad en la elección del Congreso. Un estudio empírico.

Carmen GonzÁlez EnRIQuez: Sistemas electorales y estabilidad politica en Europa Central y Oriental.

RECENSIONES. NOTICIAS DE LIBROS

PRECIOS DE SUSCRIPCION ANUAL

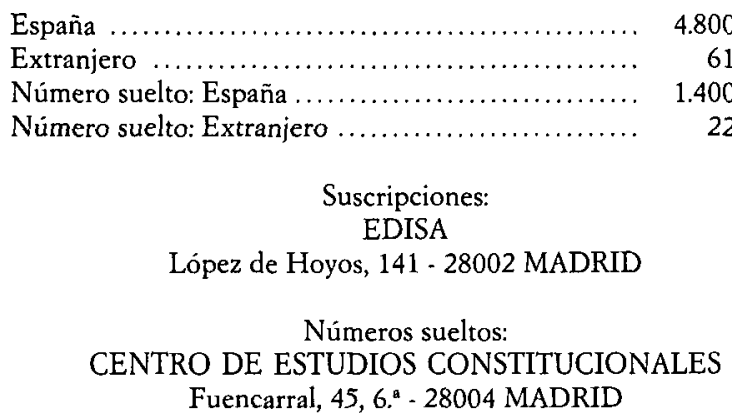




\title{
REVISTA DE ADMINISTRACION PUBLICA
}

\author{
Director: Eduardo GARCfA DE ENTERRIA \\ Secretario: Fernando SAInZ MORENo
}

SUMARIO DEL NUM. 130 (enero-abril 1993)

\section{ESTUDIOS}

F. GARRIDO FALLA: La administración única: problemática de una obviedad.

L. MARTIN-RETORTILLO BAQUER: Honorabilidad y buena conducta como requisitos para el ejercicio de profesiones y actividades.

F. LOPEZ RAMON: Reflexiones sobre el ámbito de aplicación de la Ley de Régimen Jurídico de las $A d$ ministraciones Públicas.

I. BorRajo InIESTA: La incidencia de la Ley de Costas en el Derecho urbanístico.

J. M. ALEGRE AVILA: Naturaleza y régimen jurídico de las aguas subterráneas no renovables.

A. FANLO LORAs: El control de los entes locales, ¿modelo cerrado? Reflexiones críticas sobre una dogmática consolidada.

E. GARCIA DE ENTERRIA: Un punto de vista sobre la nueva Ley de Régimen Juridico de las Administraciones Públicas y de procedimiento administrativo común.

\section{JURISPRUDENCIA}

I. Comentarios monográficos

D. V. BLANQUER C: El llamado recurso en interés de Ley: la legitimación y su fundamento extraprocesal; la postulación.

J. F. LOPEZ FONT MÁRQUEZ: El juez de instrucción como garante del derecho fundamental a la inviolabilidad del domicilio frente a la Administración.

II. Notas

- Contencioso-administrativo

A) En general (T. FONT I LlOvet y J. TORnOS Mas)

B) Personal (R. Entrena Cuesta).

CRONICA ADMINISTRATIVA

BIBLIOGRAFIA

PRECIOS DE SUSCRIPCION ANUAL

España

4.800 ptas.

Extranjero

$61 \$$

Número suelto: España

1.700 ptas.

Número suelto: Extranjero

$22 \$$

Suscripciones:

EDISA

López de Hoyos, 141 - 28002 MADRID

Números sueltos:

CENTRO DE ESTUDIOS CONSTITUCIONALES

Fuencarral, 45 - 28004 MADRID

CENTRO DE ESTUDIOS CONSTITUCIONALES

Plaza de la Marina Española, 9

28071 MADRID 


\title{
REVISTA ESPAÑOLA DE DERECHO CONSTITUCIONAL
}

\author{
Presidente: Luis SAnchez Agesta \\ Director: Francisco RUBIo LLORENTE \\ Secretario: Javier JimÉnez CAMPo
}

SUMARIO DEL AÑO 13, NUM. 37 (enero-abril 1993)

\section{ESTUDIOS}

Bartolomé CLAVERo: "Territorios Forales»: Página española del Palimpsesto.

Antonio EMBID IRUjo: Las competencias constitucionales y estatutarias sobre las aguas continentales, planteamiento normativo y realidad jurídica.

Ramón PUNSET BlanCo: La territorialización del Senado y la reforma de la Constitución.

María Rosa Ripollés SeRrano: La funcionalidad del Senado en el Estado de las Autonomías.

Mario D. SERRAFERO: El Congreso de la nación argentina y los proyectos de reforma constitucional.

Rubén HeRnÁndez VALLE: El poder constituyente derivado y los límites jurídicos del poder.

NOTA

Udo STEINER: La controversia constitucional en Alemania sobre la regulación penal del aborto.

\section{JURISPRUDENCIA}

Joaquín GaRCta MURCLA: Jurisprudencia constitucional en materia de Seguridad Social: la protección del desempleo.

Rafael Bustos Gisbert: Competencias legislativas recurrentes, garantía del cumplimiento del Derecho comunitario y ejecución interna del Derecho privado europeo.

Crónica, por el Departamento de Derecho Consttrucional de la Universidad Carlos III de MADRID.

Crónica parlamentaria, por Nicolás Pérez-SerRano Jáuregur.

\section{CRITICA DE LIBROS}

Luis Maria Diez-PicAzo: La formación de un derecho administrativo europeo.

Manuel Medina Guerrero: Föderalismus und Integrationsgewalt.

Ana Victoria SÁnchez URRUtia: La fuerza de la Constitución y la Constitución de la fuerza.

RESEÑA BIBLIOGRAFICA. Noticias de libros. Revista de revistas.

\section{PRECIOS DE SUSCRIPCION ANUAL}

España ..................................

Extranjero 4.500 ptas.

Número suelto: España

Número suelto: Extranjero

Suscripciones: EDISA

López de Hoyos, 141 - 28002 MADRID

Números sueltos: 


\title{
REVISTA DE INSTITUCIONES EUROPEAS
}

\author{
Directores: Manuel Diez de Velasco, Gil Carlos Rodriguez Iglestas \\ y Araceli Mangas Martin \\ Directora ejecutiva: Araceli MANGAS MaRTIN \\ Secretaria: Nila TORRES UGENA
}

SUMARIO DEL VOL. 20, NUM. 1 (enero-abril 1993)

\section{ESTUDIOS}

Carlos B. Jiménez PIERNAS: La protección consular y diplomática del ciudadano de la Unión Europea.

Concepción EsCoBAR HeRnANDEZ: El Convenio de aplicación de Schengen y el Convenio de Dublin: una aproximación al asilo desde la perspectiva comunitaria.

Javier RoldÁn BaRBero: Democracia y Derecho europeo.

\section{NOTAS}

Luis Ignacio SAnChez RodRIGuez: Derecho Comunitario y Derecho del mar. (Observaciones a la sentencia del TJC de 26 de noviembre de 1992.)

Alejandro VALLE GAlvez: La especificidad del ordenamiento comunitario. (Comentario a los Dictámenes 1/91 y 1/92 del TJCE sobre el Espacio Económico Europeo.)

Ernesto GaRCia TREvijano: Sobre la incorporación del Derecbo Comunitario en el Derecbo interno: una visión práctica.

Luis Miguel Hinojosa MARTINez: Los precios predatorios y el Derecho de la competencia europea. (Comentario a la sentencia del TJCE de 3 de julio de 1991, as. 62/68 AKZO cbemie B V c. Comisión.)

Luis Alberto Marco AlCALA: El declive de la doctrina del origen común de las marcas tras el caso "Hag II». (Comentario a la sentencia del TJCE de 17 de octubre de 1990, as. C-10/89,SA. CNL.Sucal v. $H a G$, «Hag II».)

\section{JURISPRUDENCIA}

\section{CRONICAS}

Consejo de Europa. Comité de Ministros, por Nila Torres.

\section{BIBLIOGRAFIA}

\section{DOCUMENTACION}

PRECIOS DE SUSCRIPCION ANUAL

España 4.500 ptas.

Extranjero

$59 \$$

Número suelto: España

1.600 ptas.

Número suelto: Extranjero

$20 \$$

Suscripciones:

EDISA

López de Hoyos, 141 - 28002 MADRID

Números sueltos:

CENTRO DE ESTUDIOS CONSTITUCIONALES

Fuencarral, 45, 6." - 28004 MADRID 


\section{EL TRIMESTRE $\mathrm{E}$
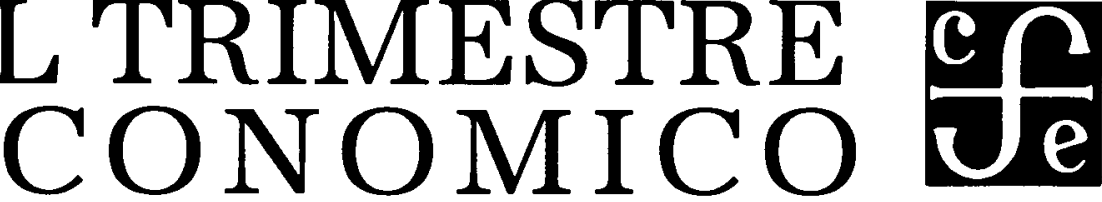

COMITÉ DICTAMINADOR: Carlos Bazdresch P., Jorge Cambiaso, Benjamin Contreras, Carlos Márquez, José Romero, Lucía Segovia, John Scott, Rodolfo de la Torre. CONSEJO EDITORIAL: Edmar L. Bacha, José Blanco, Gerardo Bueno, Enrique Cárdenas, Arturo Fernández, Ricardo FtrenchDavis, Enrique Florescano, Roberto Frenkel, Ricardo Hausmann, Albert O. Hirschman, David Ibarra, Francisco Lopes, Guillermo Maldonado, José A. Ocampo, Luis Ángel Rojo Duque, Gert Rosenthal, Fernando Rosenzweig ( $\dagger$ ), Francisco Sagasti, Jaime José Serra, Jesús Silva Herzog Flores, Osvaldo Sunkel, Carlos Tello, Ernesto Zedillo.

Director: Carlos Bazdresch P. Subdirector: Rodolfo de la Torre Secretario de Redacción: Guillermo Escalante A.

Vol. LX (1) México, Enero-Marzo de 1993

Núm. 237

\section{ARTÍCULOS}

Simón Teitel

Raúl de Gouvea Neto

y Geraldo M. Vasconcellos

Sherman Robinson, Mary E.

Burfisher, Raúl Hinojosa-Ojeda

y Karen E. Thlertelder

Rogelio Arellano Cadena

José I. Casar

Beatriz Arméndariz de Aghion
Comparación internacional entre patentes, gastos en ID, dimensión del pa/s e ingreso per capita

La diversificación de las exportaciones y la eficiencia de la cartera de exportación: Estudio comparativo de los paises del Sureste do Asia y de la América Latina

Las politicas agricolas y la migración en un área de libre comercio de los Estados Unidos y México: Un análisis de equilibrio general computable

Relación de largo plazo del mercado bursátil mexicano con el estadunidense: Un análisis de cointegración

La competitividad de la industria manufacturera mexicana. 1980-1990

El precio de los bonos, las razones deuda-exportación y las moratorias en el servicio de la deuda exterior de un pa/s: El caso de México

\section{DOCUMENTOS:}

Declaración final del Consejo de Interacción. Discurso de Miguel Mancera en la recepción del Premio de Economia Rey Juan Carlos. Gabriel Castañeda, Santiago Levy, Gabriel Martínez y Gustavo Merino, Antecedentes económicos para una Ley Federal de Competencia Económica. Ley Federal de Competencia Económica

Precio de suscripción por un año, 1993

La suscripción en México cuesta $\$ 90,000, N \$ 90,00$

Personal

$$
\begin{gathered}
\text { España, Centro } \\
\text { y Sudamérica } \\
\text { (dólares) }
\end{gathered}
$$

Universidades, $\$ 30.00$

Resto del mundo (dólares)

$\$ 42.00$ 


\title{
Rivista di Politica Economica
}

Direttore responsabile: MARIO BALDASSARRI

Direttore: INNOCENZO CIPOLLETTA

\author{
S O M M A R I O \\ IL CICLO ECONOMICO: \\ TEORIA, EVIDENZA EMPIRICA E POLITICHE CONGIUNTURALI

\section{INTRODUZIONE}

Paolo Annunziato - Mario Baldassarri

\section{LE FLUTTUAZIONI DEL CICLO ECONOMICO}

Marco LIPPI: Sulla discussione recente a proposito de teoria e misuracione del ciclo economico. Paolo Onofri - Paolo Paruolo - Bruno Salrturo: Sulle fonti delle fluttuazioni delleconomia italiana: una analisi con sistemi VAR strutturali.

Stefano FaChin - Andrea Gavosto - Guido Pellegrini: Nuove misure della componente permanente della produzione industriale: un confronto a piu paesi.

Giuseppe SCHLITZER: La teoria del ciclo economico reale.

\section{GLI INDICATORI CICLICI E L'ANALISI CONGIUNTURALE}

Paolo ANNUNZIATO: L'uso degli indicatori ciclici nell'analisi congiunturale.

Ronny NiLsson: Gili indicatori di previsione dei paesi Ocse e dell Europa centrale e orientale. Enrico GIOVANNINI: Un modello mensile del settore industriale per l'analisi del ciclo economico.

Pietro GENNARr: Analisi congiunturale e previsioni di produzione industriale.

Innocenzo Cipolleta: Morte e rinascita del ciclo economico in lialia.

Direzione, Redazione, Amministrazione: Viale Pasteur, 6 - 00144 Roma

Abbonamento annuo: Italia: L. 170.000 - Estero: L. 220.000 - Un numero L. 20.000 


\title{
CUADERNOS DE ECONOMIA
}

Publicación del Consejo Superior de Investigaciones Cientificas, en colaboración con el Departamento de Teoria Económica de la Universidad de Barcelona

Director: Joan HORTALA I ARAU

Consejo de Redacción: Cristina CARRASCO BENGOA, Juan FERnÁNDEZ DE CASTRO Rivera, Jesús Fresno LOZANo (Secretario), Juan TUgores QUES Secretaria: Gonzalo Bernardos Domínguez, Agusti Colom Cabau

\author{
SUMARIO (Vol. 20, n. ${ }^{\circ}$ 57/58) \\ III JORNADAS DE ECONOMIA CRITICA \\ Presentación \\ Tema de debate: \\ EL MERCADO: ¿MITO, ENTELEQUIA O REALIDAD?
}

David ANISI: Vino nuevo en odres viejos.

Federico AguiLera: Algunas reflexiones sobre la imposibilidad de separar la jerarquia, el mercado y los valores.

Mikel Gómez Uranga - Miguel Sánchez Padrón: Comentarios a la ponencia de David Anisi.

Félix OVEJERo LuCAS: La economía como ciencia, el mercado como moral.

\section{SELECCION DE PONENCIAS PRESENTADAS EN LAS JORNADAS}

Jesús Albarracín - Pedro Montes: El estado de la crisis económica y los interrogantes de la salida.

Cristina CARRASCO: El trabajo de las mujeres: producción y reproducción (algunas notas para su reconceptualización).

Carlos CASTILla GuTiérRez: ${ }_{2}$ Puede la valoración del medio ambiente resolver el problema de su gestión eficaz?

Oscar de JUAN: El desempleo como resultado normal del funcionamiento noral de mercados normales.

Ramón FERNÁNDEZ DURÁN: Implicaciones espaciales del quinquenio de la euforia (1986-90).

Miguel Angel GarCia CaLAvia: Flexibilidad y mercado laboral en el sector del comercio. El caso de Compredona.

José IGLESIAS: Renta minima de inserción: un caso de beneficencia pública.

Pere MIR: Las bases ingenieriles de la función de producción.

Xoxe Luis OUTES Ruso: Ondas largas e innovación. Un comentario a recientes aportaciones.

Albert RECIO: El desempleo eficiente (un paseo poco respetuoso por algunas explicaciones teóricas del paro).

LISTA DE PONENCIAS PRESENTADAS A LAS JORNADAS.

RELACION DE PERSONAS INSCRITAS A LAS JORNADAS.

\section{SUSCRIPCIONES:}

Se dirigirán a la Secretaria de la Revista (Av. Diagonal, 690 - 08034 Barcelona. Tel. (93) 402 1937, rigiendo las siguientes condiciones de venta para la suscripción anual: España, 2.500 ptas. Extranjero, 50 dólares. 


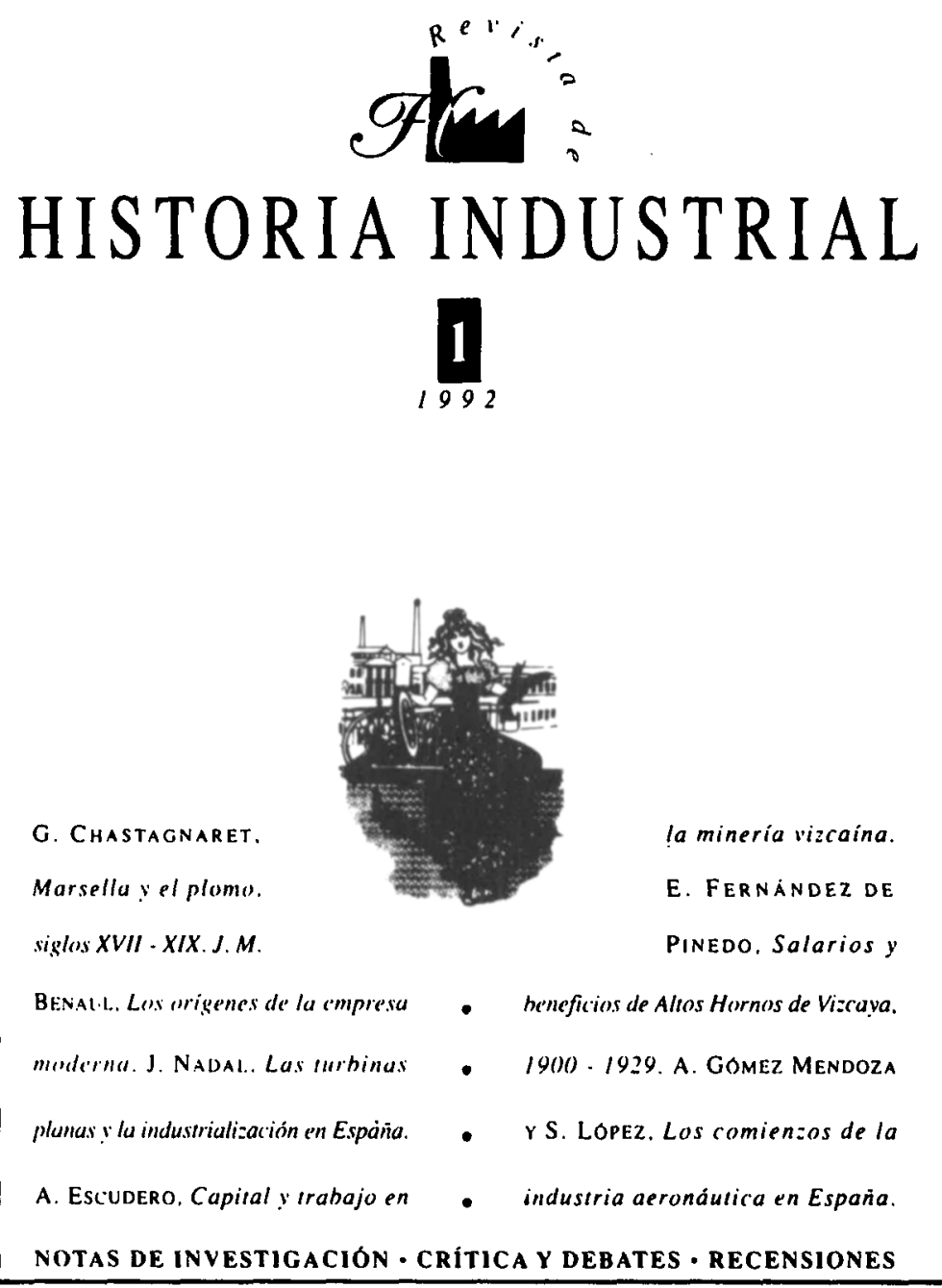

EDITA: Departament d'Història i Institucions Econòmiques (Universitat de Barcelona).

DiRECTOR: Jordi Nadal i Oller.

Consejo de Redacción: Joan Carmona Badía, Albert Carreras i Odriozola, Emiliano Femández de Pinedo, Antonio Gómez Mendoza, Jordi Maluquer de Motes, Antonio Parejo Barranco, Pere Pascual i Domènech, Carles Sudrià i Triay, Jaume Torras i Elias.

SeCRETARIO: Alejandro Sánchez Suárez.

SeCretaría de la Revista y Recepción de artículos:

Departament d'Història i Institucions Econòmiques. Facultat de Ciències Econòmiques i Empresarials. Universitat de Barcelona. Avda. Diagonal, 690 - 08034 Barcelona. Tel. 93 - 2805161 - Fax. 2802378. 


\title{
ASOCIACION INTERNACIONAL DE HISTORIA ECONOMICA
}

\author{
XI CONGRESO INTERNACIONAL
}

Universidad Bocconi - Milán - 12-17 septiembre 1994

PROGRAMA PROVISIONAL (Sesiones A y B)

\section{SESIONES A}

A1. La empresa global: Las grandes firmas y la riqueza de las naciones en la última centuria, 1890-1990.

Coordinadores: A. D. Chandler, Jr. (USA)

P. FRIDENSON (Francia)

F. AMATORI (Italia)

A2. Los problemas de la transición a la economia de mercado.

Coordinador: I. BEREND (USA-Hungria)

A3. Las relaciones cambiantes entre las regiones europeas. Division y cooperación. Siglos XIV-XVIII. Coordinador: A. MACZAK (Polonia)

\section{SESIONES B}

B1. Los poderes públicos y la producción económica en la antigiiedad clásica.

Coordinadores: J. ANDREAU (Francia)

P. ORSTED (Dinamarca)

B2. El desarrollo de la energía eléctrica. Comparaciones internacionales (1880-1980).

Coordinadores: A. BELTRAN (Francia)

P. HERTNER (Italia)

H. MORSEL (Francia)

B3. Las redes de comunicaciones europeas (siglos XIX $y X X$ ). Nuevos enfogues para el estudio de un sistema transnacional de transporte $y$ comunicación.

Coordinadores: A. CARreras (España)

A. GIUNTINI (Italia)

M. MERGER (Francia)

B4. El control de las aguas en Europa (siglos XII-XVI).

Coordinadores: E. CROUZET-PAVAN (Francia)

J. C. Viguier (Italia) 
B5. Consecuencias socioeconómicas de los coeficientes sexuales ("sex ratios») en perspectiva histórica.

Coordinadores: A. FAUVE-ChamouX (Francia)

S. SOGner (Noruega)

A. Eiras Roel (España)

B6. La integración del mercado internacional de trabajo y el impacto de las migraciones sobre los mercados nacionales de trabajo desde 1870.

Coordinadores: T. J. HATTON (RU)

J. Williamson (USA)

B7. La evolución estructural del sistema económico del Extremo Oriente desde 1700.

Coordinadores: H. KaWaKaTSU (Japón)

A. J. H. LATHÁN (RU)

B8. Trabajo y ocio en perspectiva bistórica.

Coordinadores: I. BLANCHARD (RU)

B. N. MIRÓNOV (URSS)

B9. Crecimiento económico y cambio estructural. Enfoques comparativos a largo plazo basados en series de renta nacional.

Coordinadores: A. MADDison (Holanda)

H. VAN DER WEE (Bélgica)

B10. Inversión extranjera en América Latina: sus efectos sobre el desarrollo económico, 1850-1930.

Coordinador: C. MARICHAL (México)

B11. La economia política del proteccionismo y el comercio, siglos XVIIJ.XX.

Coordinadores: J. V. C. NYE (USA)

P. LINDERT (USA)

B12. La evolución de las instituciones financieras modernas.

Coordinadores: U. Olsson (Suecia)

G. D. FeldMan (USA)

B13. La nación, Europa y el mercado en el pensamiento económico.

Coordinadores: P. RoGGI (Italia)

L. BAECK (Bélgica)

G. GıOLI (Italia)

B14. Los salarios reales en los siglos XIX $y X X$.

Coordinadores: V. ZAMAGNI (Italia)

P. SCHOLliers (Bélgica)

B15. La cultura material: consumo, estilo de vida, nivel de vida (1500-1900).

Coordinadores: A. J. SCHUURMAN (Holanda)

L. S. WALSH (USA)

B16. Gestión, finanzas y relaciones industriales en la industria maritima.

Coordinadores: S. P. VILLE (Nueva Zelanda)

D. M. WILliams (RU)

Aunque el orden, titulo y coordinadores de las sesiones son provisionales, los interesados pueden dirigirse a los coordinadores, a la Secretaria de la Asociación Internacional (Prof. Josep GoY, Sécrétaire Gènèral, Ecole de Hautes Etudes en Sciences Sociales, Centre de Recherches Historiques, 54 Blvd. Raspail, 75270 Paris CEDEX 06, Francia), o a la Secretaria de la Asociación Española (Prof. Pablo Martín ACEÑa, Facultad de Ciencias Económicas y Empresariales, Universidad de Alcalá, Plaza Victoria, 3, Alcalá de Henares, Madrid). 


\title{
XI CONGRESO INTERNACIONAL DE HISTORIA ECONOMICA
}

\author{
Milán, Septiembre 1994 \\ TEMAS C (Seminarios)
}

Espacio urbano y organización económica en la Europa medieval (Alberto Grobman, Italia).

The role of crafts and craftsmen in ancient near eastern economies (Johannes Renger, Universidad de Berlin).

Tenurial relations and markets in late medieval and early modern Europe (Epstein, Trinity College, Cambridge, UK).

The Florentine economy and east-central Europe in the 14th and 15th centuries(Susanna Teke, Hungarian Academy of Sciences, Budapest).

Organisation commerciale et économie régionale dans l'éspace alpin, $\mathrm{xV}^{*}$-xVIII siècles (Christian Pfister, Universidad de Zurich).

Plague and trade in Europe and North Africa during the early modern period (15th-18th centuries) (Gonfal López Nadal, Universitat de las Illas Balears, Mallorca).

La base pré-statistique pour les recherches socio-démographiques en Europea XVI-XVIII siècles (Androjei Wyczanski, Academia Polaca de Ciencias, Varsovia).

Information technology and transaction costs in the development of firms, markets, and economies (James Foreman-Peck, St. Antony-s College, Oxford).

Le financement de l'entreprise au fil de l'industrialisation (milieu $\mathrm{xvIII}^{\mathrm{c}}$-milieu $\mathrm{xx}^{\mathrm{c}}$ siècles) (Alain Plessis, París).

Competition and cooperation of enterprises on national and international markets (19th-20th centuries) (Hans Pobl, Universidad de Bonn).

International cartels revisited (Kudo, Asahigaoka 1.615.3, Kiyoshe-shi, Tokyo 204).

L'entreprise privée en période de crise économique: stratégies de survie ou stratégies de renouvellement? (Lanthier, Université de Quebec à Trois Rivières, Trois Rivières, Canadá).

The firm and the businessman in capitalist economies (Robertson, The University of New South Wales, Canberra).

The free-standing company within the international economy, 1870-1970 (Harm Schroeter, Freie Universitaet Berlin).

Capital flows and entrepreneurial strategies in Southern Europe and the Balkans (19th and 20th centuries) (George Dertilis, Universidad de Atenas).

Agricultural labour: genesis, forms of employment, changing role in agricultural production (Grigory Kotovsky, Academy of Sciences, Moscú).

Agrarian technology in North-west Europe in the Middle Ages. Developments and comparisons ((Langdon, History, University of Alberta, Canadá).

Nécessités économiques et pratiques juridiques: problèmes de la transmission des exploitations agricoles, XVII'-XX' siècles (Joseph Goy, EHESS-CRH, 54 Bd Raspail, 75270 París).

An international view of commercialization in agriculture (Marvin McInnis, Queens University, Canadá).

Technological change and the labour process in the sugar industry, 1815-1914 (Bill Albert, University of East Anglia, UK). 
Industry and design since the Industrial revolution (Loy Church, University of East Anglia, UK).

Film: an industry on the crossroads of economics, politics, and art (Klimes, Cesky Filmovsky Ustav, Praga).

Skill formation for industry: Europe, USA, and Japan (Reiko Okayama, Meiji University, Tokyo 101).

The social organization of iron production in Europe, 1600-1900 (Goran Ryden, Universidad de Uppsala, Suecia).

Forced labour and labour markets, historical approaches (Herman Diederiks, Universidad de Leyden).

The emergence of a transatlantic labor market in the nineteenth century: confronting the North and the South experiences (Fernando Devoto, Centro de Estudios Migratorios Latinoamericanos, Independencia 20, Buenos Aires).

Production and consumption of beer sonce 1500 (Erik Aerts, Vlaamse Ekonomische Hogeschool, Koningsstraat 336, 1210 Bruselas).

Comparative history of European stock exchanges (Ranald Michie).

International banking in the northern Pacific area, 1859-1959 (Tamaki, Keio University, Tokyo 108).

Trade and pre-colonial commercial structures of the Indian sub-continent Uoseph, Mysore University, Mysore 570006, India).

Saharian business and merchant capital in Nineteenth-century international commerce (McDougall, University of Alberta, Canadá).

Commercial networks in Asia, 1850-1959 (Suguyama, Keio University, Tokyo 108).

Oceanic trade, colonial wares and industrial development, 1600-1800 (Maxine Berg, UK).

Micro-et macroéconomie de la protection sociale (de l'Antiquité à nos jours) (Gueslin, Université Blaise Pascal, 63037 Clermont-Ferrand, Francia).

The political economy of late-nineteenth century government regulation (/Gary Libecap, University of Arizona, USA).

Food policy during the World wars in the Twentieth century (Oddy, The Polytechnic of central London, UK).

The system of centrally planned economies in central-east and south-east Europe after World War II and the causes of its decay (Vaclav Pruca, Prague School of Economics, Checoslovaquia).

Creating local-government infrastructure in the industrialization process: a financial and budgetary perspective (Richard Tilly, Universitaet Muenster, Alemania).

Economic associations and political change in late Nineteenth-century Europe (Paola Subacchi, Università Bocconi, Milán).

Croos-country comparisons of industrialization in small countries, 1870-1940: attitudes, organizational patterns, technology, productivity (Olle Krantz, Umea University, S90187 Umea, Suecia).

Transport et crissance del économies africaines aux $\mathrm{XIX}^{\bullet}$ et $\mathrm{XX}^{\bullet}$ siècles (Tsund Olela, B. P. 4749, Lubumbashi, Zaire).

Strategies for developing and exploiting new technologies: USA and Japan (André Millard, University of Alabama at Birmingham, USA).

Diffusion of technology and European integration, 1840-1914 (Kristine Bruland, Universidad de Osio).

Colonialism and technology choices (Dwijandra Tripathi, Indian Institute of Management, Ahmadabad 380056, India). 
Trajectoires individuelles (aspects démographiques et sociaux) dans une période de mutations économiques (Bourdelais, EHESS-CRH, 54 Bd Raspail, 75270 Paris).

Nineteenth and Twentieth-century Business Cycles: the interplay of historical data, reconstruction, and analysis (Trevor Dick, University of Lethbridge, Canadá).

Histoire des télecommunications (Griset, IHMC, 45 re d'Ulm, 75005 Paris).

Economic history and the arts (Michael North, Universitaet Kiel, Alemania).

Japan's war economy (Eric Pauer, Philipps Universitaet Marburg, Alemania).

Cities at war, 1914-1918 (Jay Murray Winter, Pembroke College, Cambridge CB2 1RF, UK).

Production networks: market roles and social norms (Carlo Poni, Università di Bologna).

Coastal communities in a cross-cultural and historical perspective: the interaction of economic activity and societal change (John Rogers, Uppsala University, Uppsala, Suecia).

Recent developments in cliometrics (Sam Williamson, Miami University, Oxford, Ohio 45056, USA). 


\title{
INTERNATIONAL ECONOMIC HISTORY ASSOCIATION
}

\author{
Eleventh International Economic History Congress
}

\author{
SESSIONS D \\ COMPETITION FOR RECENTLY COMPLETED DOCTORAL THESES
}

Young scholars are invited to present summaries of their doctoral research at the Milan Congress of the International Economic History Association in September 1994. Summaries of these theses will be published in a volume of the congress proceedings, and four diploma and four prizes of 1.000 dollars will be awarded.

To be eligible for these sessions candidates must have been awarded their doctorate or equivalent after 1 September 1988 and not later than 31 December 1992.

Scholars interested in participating in these sessions should write details without delay to:

\author{
Professor Joseph GoY \\ General Secretary \\ International Economic History Association \\ Centre de Recherches Historiques \\ 54 Bd Raspail \\ 75270 Paris Cedex 06 - France
}

They should specify the thesis's title, supervisor and assessors, and the institution which awarded the degree. Please do not send thesis. 


\section{ALIANZA EDITORIAL}

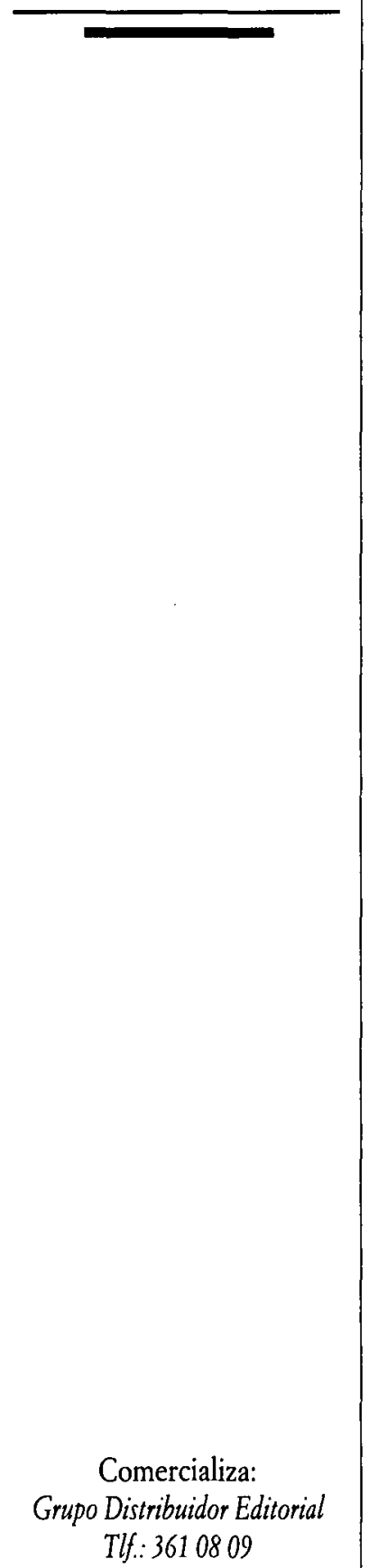

\section{ALIANZA UNIVERIDAD}

Alfonso Botti

CIELO Y DINERO

El nacionalcatolicismo

en España (1881-1975)

AU 717

\section{Charles Tilly}

COERCION, CAPITAL Y LOS

ESTADOS EUROPEOS,

990-1990.

AU 721

S. Bowles, D. M. Gordon

y T.E. Weisskopf

TRAS LA ECONOMIA DEL

DESPILFARRO

Una economía

democrática para

el año 2000

AU 735

Leandro Prados de la

Escosura y Samuel

Amaral (Editores)

LA INDEPENDENCIA

AMERICANA:

CONSECUENCIAS

ECONOMICAS.

AU 745

Pablo Fernández

Albaladejo

FRAGMENTOS DE

MONARQUIA

AU 734

\section{Thomas F. Glick}

TECNOLOGIA, CIENCIA Y

CULTURA EN LA ESPAÑA

MEDIEVAL

AU 725

Joel Mockyr

LA PALANCA DE LA RIQUEZA.

Creatividad tecnológica y progreso económico.

AU 748

\section{EL LIBRO DE BOLSILLO}

Chris Cook

DICCIONARIO DE

TERMINOS HISTORICOS.

LB 1602

\section{ALIANZA ECONOMIA}

\section{Robert M. Solow}

EL MERCADO DE TRABAJO

COMO INSTITUCION

SOCIAL

AE 2

Antonio Espasa y José

Ramón Cancelo

(Editores)

METODOS CUANTTTATTVOS

PARA EL ANALISIS DE LA

COYUNTURA ECONOMICA.

AE 3 


\section{NUEVO DICCIONARIO BILINGÜE DE ECONOMIA $Y$ EMPRESA Inglés-español Español-inglés}

José María Lozano Irueste

$3^{a}$ edición revisada y ampliada Cartoné, $21 \times 26,5 \mathrm{~cm}, 896$ páginas
El Nuevo Diccionario Bilingüe de Economía y Empresa, único en la bibliografía española, ofrece una recopilación exhaustiva de términos y expresiones del lenguaje economico-empresarial, pertenecientes, tanto a la economía en todas sus ramas $y$ de las ciencias próximas a ellas, como las del léxico común que se utiliza con frecuencia en los textos económicos. Consta de dos partes: la primera, inglés-español, ofrece 61.808

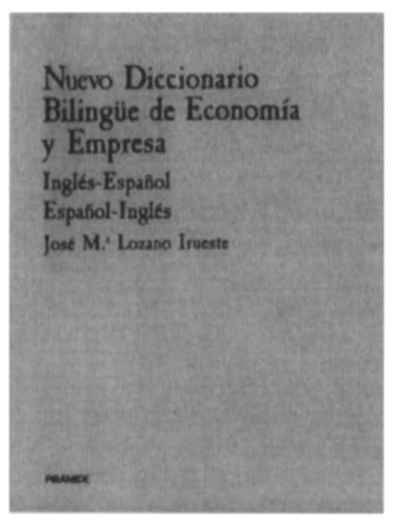

\section{EL SISTEMA JUST IN TIME Y LA FLEXIBILIDAD DE LA PRODUCCIÓN}

Tomás M. Bañegil

Rústica, $14 \times 22 \mathrm{~cm}, 264$ páginas

Presenta una reflexión actual, sistemática y detallada de todos los conceptos teóricos y prácticos que sobre la flexibilidad de la producción en general, y del sistema just in time (JIT) en particular, existen actualmente. ¿Es el JIT la causa principal del éxito japonés? ¿Está siendo adaptado correctamente por la industria española? ¿Es el JIT un buen sistema de trabajo en los actuales momentos de crisis?

\section{LA NUEVA DIRECCIÓN DE PERSONAS. MARCO PARADÓJICO DEL TALENTO DIRECTIVO} José María Gasalla

Rústica, $14 \times 22 \mathrm{~cm}, 352$ páginas

Sugerente $e$ insistentemente, analítica y científicamente, conjugando el rigor y el pensamiento creativo, el autor nos invita a redescubir el talento directivo necesario para mejorar la eficacia en la dirección de personas en el marco paradójico en el que se mueven las organizaciones actuales.
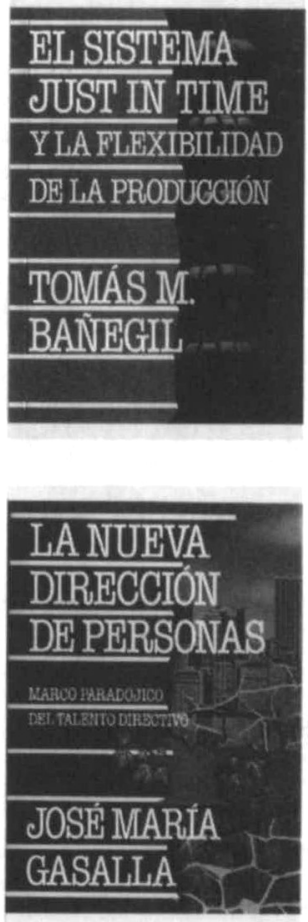

\section{SPIRAMIDE}

Telémaco, 43. 28027 Madrid

Tel. (91) 32001 19. Fax 7426631
Comercializa:

Grupo Distribuidor Editorial, S. A. Ferrer del Rio, 35. 28020 Madrid Tel. (91) 3610809 . Fax 3565702 


\section{CENTRO DE ESTUDIOS CONSTITUCIONALES}

\section{ULTIMAS PUBLICACIONES}

Manuel Garcia Pelayo: Obras completas ( 3 tomos). 18.500 ptas.

Constitución Española, 1978-1988. Obra dirigida por Luis Aguiar de Luque y Ricardo Blanco Canales ( 3 vols.). 35.000 ptas.

Paloma Biglino Campos: Los vicios en el procedimiento legislativo. 1.400 ptas.

C. McIlwain: Constitucionalismo antiguo y moderno. Traducción de Juan J. Solozábal. 2.200 ptas.

ANTONIO Fanlo LoRAS: Fundamentos constitucionales de la Autonomia Local. 3.700 ptas.

Pablo Salvador CoDerch y otros: El mercado de las ideas. 3.400 ptas.

Javier Pardo Falcón: El Consejo Constitucional Francés. 3.500 ptas.

ANDRÉs BeTANCOR: El Acto Ejecutivo. 2.700 ptas.

ANGEl Gómez MONTORO: Conflictos de atribuciones entre órganos del Estado. 3.000 ptas.

Jesús Prieto de Pedro: Cultura, culturas y Constitución. 2.700 ptas.

MANUEl MEDINA GUERRERO: La incidencia del sistema de financiación en el ejercicio de las competencias de las CC.AA. 3.300 ptas.

KonRAD Hesse: Estudios de Derecho Constitucional (2.a ed.). 1.100 ptas.

Fulvio ATTINA: Introducción al sistema politico de la Comunidad Europea. 1.700 ptas.

Centro de Estudios Institucionales De Buenos Aires: El presidencialismo puesto a prueba. 2.400 ptas.

Elide KeDourie: Nacionalismo. Prólogo de Francisco Murillo Ferrol. Traducción de Juan J. Solozábal Echevarría. 900 ptas.

RAMÓN GARCia COTARELO: Del Estado del bienestar al Estado del malestar. 1.800 ptas.

Alfonso Ruiz Miguel: La justicia de la guerra y de la paz. 2.000 ptas.

GREgorio PECES-BARBA: La elaboración de la Constitución de 1978. 2.000 ptas.

Pilar Chávarri Sidera: Las elecciones de diputados a Cortes Generales y Extraordinarias (18101813). 2.200 ptas.

ALF Ross: ${ }_{i}$ Por qué Democracia? 1.500 ptas.

ANGEl RoDriguez Diaz: Transición politica y consolidación constitucional de los partidos politicos. 1.600 ptas.

MANUEl Ramírez: Sistema de partidos politicos en España (1931-1990). 1.700 ptas.

Javier Corcuera Atienza: Politica y Derecbo. La construcción de la Autonomia vasca. 2.300 ptas.

José María Garcia Marín: Monarquia católica en Italia. 2.800 ptas.

Antonio Serrano GonzÁlez: Como lobo entre ovejas. Soberanos y marginados en Bodin, Shakespeare, Vives. 2.500 ptas.

Jesús VALLejo: De equidad ruda a ley consumada. Concepción de la potestad normativa 1250-1350. 2.800 ptas.

José Maria PoRtillo Valdés: Monarquia y gobierno provincial. Poder y Constitución en las provincias vascas (1760-1808). 3.600 ptas.

BARTOLOME Cla vero SALVADOR: Razón de Estado, razón de individuo, razón de historia. 1.800 ptas.

CARMen MUNoz De Bustillo Romero: Bayona en Andalucia: El estado bonapartista en la prefectura de Xerez. 2.800 ptas.

JERÓNIMO BETEGON: La justificación de castigo. 2.700 ptas.

JOSÉ MARTÍNEZ DE PISÓN: Justicia y orden politico en Hume. 2.600 ptas.

MARTIN D. FARRELL: La filosofia del liberalismo. 2.300 ptas.

Carlos Thiebaut: Los limites de la Comunidad. 1.800 ptas.

EMILIO LLEDÓ: El silencio de la escritura. 800 ptas.

AULIS AARNIO: Lo racional como razonable. 2.200 ptas.

RAFAel DE Asís RoIG: Deberes y obligaciones en la Constitución. 2.800 ptas. 
M." Teresa Rodríguez DE Lecea: Antropología y filosofía de la bistoria en Julian Sanz del Rio. 1.700 ptas.

MARINA GASCÓN ABELLÁN: Obediencia al derecbo y objeción de conciencia. 2.600 ptas.

JEAN LOUIS DE LOLME: Constitución de Inglaterra. 2.500 ptas.

JoAQuín Costa: Historia critica de la Revolución Española. 2.600 ptas.

Gaspar DE ANASTRo Isuza: Los seis libros de la República de Bodino traducidos del francés y católicamente enmendados. Ed. preparada por José Luis Bermejo. 6.000 ptas.

Francisco Murillo Ferrol: Saavedra Fajardo y la política del Barroco. 2." edición. 1.800 ptas.

JuAn ROMERo Alpuente: Historia de la Revolución española y otros escritos. Edición preparada e introducida por Alberto Gil Novales. Dos volúmenes. 5.000 ptas.

JOSÉ MARCHENA: Obra española en prosa. 1.700 ptas.

Juan Maldonado: El levantamiento de España. Edición bilingüe. Traducción e introducción de M.a Angeles Durán. 3.600 ptas.

HobBes: Bebemoth. Traducción e introducción de Antonio Hermosa Andújar. 2.500 ptas.

Guillermo Occam: Obra Politica. Traducción de Primitivo Mariño. 3.700 ptas.

ARISTÓteles: Política. Edición bilingüe. Reimpresión. 1.800 ptas.

ARISTÓteles: Etica a Nicómaco. Edición bilingüe. Reimpresión. 1.200 pras.

ARISTÓTELES: Retórica. Edición bilingüe. Reimpresión. 1.800 ptas.

Senac De Meilhan y A. Barnave: Dos interpretaciones de la Revolución Francesa. 1.600 ptas.

Tomás de Campanela: La Monarquia del Mesias y las Monarquias de las Naciones. Traducción e introducción de Primitivo Mariño Gómez. 1.800 ptas.

JuAn Altusio: La política. 4.800 ptas.

J. Bentham: Falacias politicas. 2.200 ptas.

E. SIEYĖs: Escritos y discursos de la revolución. 2.200 ptas.

G. JELLINEK: Reformas y mutación de la Constitución. 1.800 ptas.

CONDORCET, CASTILLÓN y BECKER: ¿Es conveniente engañar al pueblo? Traducción e introducción de Javier de Lucas. 2.300 ptas.

Plutarco: Consejos politicos. Edición bilingüe. 2.000 ptas.

Constituciones Iberoamericanas. Edición preparada por Luis López Guerra y Luis Aguiar de Luque. 4.600 ptas.

Jurisprudencia del Tribunal de Justicia de las Comunidades Europeas. Edición preparada por Manuel Diez de Velasco y Gil Carlos Rodriguez Iglesias. 4.000 ptas.

Gonzalo Menéndez PIDAL: La España del siglo XIX vista por sus contemporáneos. Dos volúmenes. 6.000 ptas. cada uno.

mario G. Losano, Antonio E. Pérez Luño y M." Fernanda Gerrero Mateus: Libertad informática $y$ leyes de protección de datos personales. 1.300 ptas.

VICENTE ESCUIN PALOP: Régimen jurídico de la entrada y permanencia de extranjeros en España. 950 ptas.

MANUel C. PALOMEQue: Los derechos laborales en la Constitución española. 1.000 ptas.

CENTRO DE ESTUDIOS INSTITUCIONALES DE BUENOS AIRES: Fundamentos y alcances del control judicial de constitucionalidad. 1.500 ptas.

LuCIANo PARejo AlFonso: Crisis y renovación en el derecho público. 1.100 ptas.

VICTORIA CAMPS y SALVADOR GINER: El interés comin. 800 ptas.

RICHARD GUNTHER: Politica $y$ cultura en España. 1.000 ptas.

JOSÉ MARfa CONTRERAS MAZARIO: La enseñanza de la religión en el sistema educativo. 1.300 ptas.

Centro de Estudios Institucionales De Buenos Aires: La Autonomia personal. 1.400 ptas.

JoAn Subirats Humet: Un problema de estilo. La formación de paliticas públicas en España. 1.300 ptas.

DANIEL MENDOZA: Introducción al análisis normativo. 1.100 ptas.

Luis Prieto Sanchis: Principios y normas. Problemas del razonamiento juridico. 1.300 ptas. 


\title{
MP \\ MARCIAL PONS \\ LIBRERO
}

\author{
Información bibliográfica
}

Exposición de libros españoles y extranjeros

Cuenta de librería

Humanidades y

C.C. Sociales

Libros Juridicos

Bárbara de Braganza, 8

28004 MADRID

Tel.: 3194250

PI. Conde del Valle de Suchil, 8 28015 MADRID

Tel.: 4484797

Fax: 3194373

Fax: 5931329

Economía y Gestión

Plaza de las Salesas, 10 28004 MADRID

Tel.: 3085649

Fax: 3086030

\section{Agencia de suscripciones:}

Publicaciones nacionales y extranjeras

Números sueltos

Colecciones atrasadas

c/Tamayo y Baus, 7

28004 MADRID

Tel.: 3194254

Fax: 3194373 


\section{Revista de Historia Económica}

